\title{
PERSPECTIVAS DE LA GOBERNABILIDAD DEMOCRATICA EN VENEZUELA
}

\author{
Michael Coppedge*
}

Venezuela, en algún momento la democracia más gobernable en América Latina, es hoy una democracia sumamente frágil. Este artículo describre la fórmula que permitió la gobernabilidad en Venezuela durante los años 1970, busca sus orígenes en la década de 1960, y explica por qué se derrumbó en los años 1980, dejando al régimen democrático en una situación peligrosa en los noventa. Esta perspectiva histórica se hace necesaria para tratar de entender las perspectivas de la gobernabilidad democrática del gobierno de Caldera, del que se espera ofrezca una alternativa a la vieja fórmula pero también será comparado con los logros del régimen anterior. Si la alternativa democrática de Caldera se considera un fracaso, muchos venezolanos se verán inclinados a reconsiderar una alternativa no-democrática. La perspectiva histórica es también útil porque aporta varias lecciones sobre otras democracias latinoamericanas. En primer lugar, y dado que la fórmula venezolana funcionó bien por un período, permite identificar los elementos que componen la gobernabilidad democrática. En segundo lugar, la crisis de gobernabilidad posterior ilumina las debilidades y puntos fuertes de una fórmula que a menudo fue considerada un modelo para otros países. Y finalmente, sólo una visión de largo plazo puede indicarnos los cambios a encarar por cualquier fórmula en un contexto social y económico dinámico. Incluso los modelos con mayor éxito en cuestión de gobernabilidad deben adaptarse para sobrevivir. Los guardianes del sistema venezolano se adaptaron demasiado poco al principio, pero quizás no demasiado tarde.

\section{ELEMENTOS DE LA GOBERNABILIDAD DEMOCRÁTICA}

La gobernabilidad se entiende mejor analizando las relaciones entre los actores estratégicos, es decir, aquellos intereses organizados con el suficiente control de algunos recursos de poder -factores de producción, miembros, cargos públicos, fuerzas armadas, autoridad moral o ideas e información- para perturbar el orden público o el desarrollo económico(1). Ya sea que ellos causen problemas, amenacen simplemente con hacerlo, o tomen ventaja de un entendimiento implícito de su potencial para perturbar el orden, son

(*) Profesor en John Hopkins University de Washington. $1993 \mathrm{a}$.

(1) Para una mayor elaboración de este enfoque de gobierno véase Coppedge los únicos actores cuyo comportamiento resulta relevante para la gobernabilidad. En general, en los regímenes democráticos latinoamericanos existen tres tipos de actores estratégicos. Algunos son actores estatales, especialmente los militares (y la policía), la burocracia permanente, y el gobierno (aquéllos que ostentan temporalmente cargos públicos y dirigen la actividad del Estado). Algunos son actores sociales -la Iglesia, las asociaciones del sector privado, los sindicatos de trabajadores, los medios de comunicación, los campesinos organizados, los movimientos indígenas, incluso guerrillas y terroristas. Por último, los partidos políticos suelen ser también actores estratégicos, aunque no actúan exclusivamente en el Estado o la sociedad, sino que intentan mediar entre ellos acudiendo a las elecciones, encabezando el gobierno y representando a la sociedad civil en el Legislativo.

Gobernabilidad sería el grado en el cual las relaciones entre estos actores estratégicos obedecen fórmulas que son estables y mutuamente aceptadas. Algunas de estas fórmulas se formalizan en leyes, tales como Constituciones, códigos de trabajo, o propuestas de representación tripartita en los consejos de administración de las empresas estatales. Muchas otras fórmulas son informales, tales como coaliciones, pactos entre partidos, o la tendencia de los gobernantes de consultar con las asociaciones del sector privado. Cuando estas fórmulas son estables y mutuamente aceptadas, la violencia se minimiza, los conflictos se resuelven pacificamente, los actores "compiten según las reglas del juego", y las interacciones construyen confianza. En resumen, reina la gobernabilidad. Cuando las fórmulas que rigen las relaciones entre los actores estratégicos no son estables y mutuamente aceptadas, se producen manifestaciones de ingobernabilidad cuando algunos actores rechazan viejas fórmulas, tratan de imponer otras nuevas, o socavan el consenso de cualquier fórmula mientras construyen su propio poder o tratan de minar el poder de otros actores. Los ejemplos de tales manifestaciones abarcan desde crisis de gabinetes, estancamiento, y fraude electoral a protestas violentas, terrorismo, y golpes militares.

\section{LA FÓRMULA VENEZOLANA: LA PARTIDOCRACIA}

Venezuela desarrolló una fórmula de gobernabilidad que funcionó extraordinariamente bien en la década de 1970. Fue una fórmula que otorgó un papel principal a los dos 
partidos más importantes -el socialdemócrata Acción Democrática (AD) y el cristiano-demócrata COPEI. Muchos venezolanos han llamado a esta fórmula partidocracia (de partido y democracia), que yo he traducido como "partyarchy"(2). Los guardianes de esta fórmula, por decirlo así, fueron los anteriores adecos y copeyanos, a quienes algunos venezolanos llamaron el "status" adecopeyano y lo que llamaré el acuerdo adecopeyano, o simplemente el acuerdo.

Los términos de la fórmula partidocrática fueron como sigue:

1. Participación inclusiva. AD y COPEI representaban a casi todos los grupos de la sociedad. La afiliación de estos dos partidos era más elevada (superior al 31 por ciento del total de votantes) que la de otro partido en cualquier otro país democrático del mundo, con las posibles excepciones de Costa Rica y Chile. Dado que la mayoría de los nomiembros eran al menos simpatizantes, estos dos partidos también compartían casi el 80 por ciento del total del voto legislativo y del 90 por ciento del voto en las elecciones presidenciales entre 1973 y 1988, incluso aunque existían docenas de partidos distintos. La organización del partido era extensiva: cada pequeña ciudad en Venezuela tenía un cuartel general de AD o de COPEI. Más aún, el liderazgo de casi practicamente todas las organizaciones de la sociedad civil (distintas a la Iglesia o a las asociaciones del sector privado) fueron elegidos en elecciones que usaban listas identificadas como AD y COPEI. Aproximadamente el 80 por ciento de las federaciones de campesinos y al menos el 60 por ciento de los sindicatos obreros estaban controlados por líderes afiliados a AD.

2. Competencia electoral. Los ciudadanos y los actores sociales no afiliados al acuerdo adecopeyano reconocían, al menos, las elecciones, cuya limpieza era motivo de orgullo, como el mecanismo legítimo para decidir quién ocuparía los cargos públicos. Las campañas electorales eran festivales cívicos que duraban casi un año, que movilizaban millones en sondeos, paradas, caravanas de coches, y mitines masivos al aire libre, siempre desbordados con la parafernalia de la campaña. La abstención nunca excedió del 12,4 por ciento antes de 1988.

3. La disciplina de partido. AD y COPEI practicaban una disciplina de hierro: los militantes de todos los niveles del partido se veían amenazados con la expulsión si desobedecían las decisiones adoptadas por el pequeño círculo central de líderes, o cogollo, a la cabeza del partido. El principio leninista de centralismo democrático se recogía explícitamente en los estatutos de AD. En consecuencia, los senadores y los diputados, los legisladores estatales y los miembros del consejo municipal se desviaban con tan poca frecuencia de la línea fijada por el partido que los líderes en el Congreso ni siquiera se molestaban en llevar la cuenta o contar los votos; sólo importaban los tamaños relativos de

(2) Este concepto está más desarrollado y ha sido contrastado con el concepto de poliarquía en mi próximo libro, Strong Parties and Lame Ducks: Presidential Partyarchy and Factionalism in Venezuela (Stanford, 1994) Este apartado resume algunos argumentos desarrollados con mayor amplitud en el capítulo segundo de este libro. los partidos. Los líderes sindicales se abstenían de apoyar huelgas cuando su partido se hallaba en el poder, y los cargos políticos de las asociaciones profesionales, gobiernos de estudiantes, federaciones de campesinos, empresas estatales, fundaciones, y la mayor parte de las organizaciones utilizaban sus posiciones para profundizar los intereses de su partido. Los dos partidos actuaban además como poderosos bloques listos para mobilizarse.

4. Concertación. Los líderes de AD y COPEI se habituaron a consultarse el uno al otro, así como a líderes de otros partidos y organizaciones sociales, siempre que aparecían temas controvertidos (Levine 1973). Políticas que concernían a la defensa, asuntos exteriores, y a la industria petrolera se tomaron usualmente por consenso, y cuando éste se hizo imposible, el intento en lograrlo apaciguó a la oposición. Los líderes de los partidos estaban ampliamente convencidos de que no debía permitirse la escalada de un conflicto hasta el punto de llegar a amenazar el régimen democrático. Aunque los conflictos se produjeran, el liderazgo siempre lo frenaba a tiempo para salvar el régimen (Tugwell 1975, Coppedge 1994b).

5. Relaciones más amplias. Los partidos también se aseguraban buenas relaciones con otros actores estratégicos los militares y el sector privado(3). A cambio de no interferir en los asuntos políticos, los gobiernos de $\mathrm{AD}$ y COPEI recompensaban a las Fuerzas Armadas con elevados salarios, ambiciosos programas educacionales, ascensos frecuentes y un equipamiento sofisticado. Las asociaciones del sector privado FEDECAMARAS, CONSECOMERCIO, y CONINDUSTRIA, mientras se mostraron con frecuencia críticas con respecto a las políticas gubernamentales, también llegaron a depender de elevados subsidios, bajos impuestos y tarifas proteccionistas. Estas asociaciones se incluían a menudo en el proceso de concertación, y se entendía que el Ministro de Finanzas sería designado tras la consulta con una o más de estas gigantescas compañías que pertenecían a las familias más poderosas del país.

La gobernabilidad venía entonces asegurada por el acuerdo adecopeyano que, dado que controlaba los partidos importantes y disciplinados con influencia sobre la mayoría de las organizaciones, tenían la autoridad para negociar con otros partidos y actores estratégicos; y el poder de apoyar los acuerdos a los que se llegaba.

\section{EL SURGIMIENTO Y DECLIVE DE LA PARTIDOCRACIA}

La fórmula que acabamos de describir fue típica de los años 1970 en Venezuela pero hoy se da en una fórmula mucho más débil. Mientras los líderes de la transición democrática de 1958 se beneficiaron en muchos aspectos de su emergente partidocracia, la fórmula no se consolidó plenamente hasta 1970. Además, la década de 1970 representa un

(3) La Iglesia, que siempre ha sido comparativamente débil en Venezuela, cesó de intervenir activamente en política a principios de los años 1960 (Levine 1973) En cuanto a las relaciones con el sector privado, véase Karl 1982. Desde un punto de vista diferente, consultar Gil Yepes 1981. 
punto máximo en la aparición y declive del ciclo de partidocracia en Venezuela.

\section{CAMBIOS Y CONSOLIDACIÓN EN LOS AÑOS 1960}

Acción Democrática había sido un partido amplio, de masas, y muy disciplinado desde su fundación en 1941, pero los otros elementos de la partidocracia no existían antes de 1958 (Martz 1966). Hasta ese año sólo se habían celebrado dos elecciones nacionales libres y con sufragio pleno en 1946 y 1948, interrumpidas por la dictadura militar de Marcos Pérez Jiménez. COPEI había surgido durante el Trienio 1945-1948 pero no como la contraparte para la concertación con AD; de hecho, los Copeyanos y la jerarquía eclesiástica habían apoyado el golpe que acabó con el primer gobierno de AD en 1948 (Levine 1978). Los militares habían sido perseguidos por $\mathrm{AD}$ durante la última década, y algunos líderes del mundo empresarial se mostraban cautelosos ante un regreso del gobierno de AD dada su orientación de centro-izquierda. Después de que Pérez Jiménez fuera derrocado por un golpe interno, las negociaciones entre $\mathrm{AD}, \mathrm{COPEI}$, URD y un líder empresarial culminaron con el Pacto de Punto Fijo de 1958, que completó los otros elementos de la partidocracia. Bajo el liderazgo de Rómulo Betancourt y Rafael Caldera, AD y COPEI formaron un frente único solicitando elecciones, lo que inició una larga tradición de concertación. Apoyándose en la disciplina del partido, AD prometía tranquilidad laboral si los líderes empresariales apoyaban las elecciones, y éstos estuvieron de acuerdo, lo que dejó a los militares sin otra posibilidad que no fuera completar la transición (Karl 1986). El Pacto comprometía expresamente a los dirigentes partidistas a usar sus organizaciones para moderar el conflicto político, y aunque Betancourt fue el claro ganador en la elección presidencial de 1958, cedió para lograr un gobierno de unidad nacional en el cual estuvieran representados por igual los tres partidos.

La fórmula que hizo posible la transición a la democracia se enfrentó con varios cambios importantes en sus primeros años pero logró reducirlos. Los militares no se mostraron completamente unidos en su apoyo a la democracia, pero el asiduo cortejo de Betancourt hacia los militares le permitió sobrevivir a cuatro intentos de golpes de estado (Alexander 1964). Los movimientos guerrilleros amenazaron a la democracia venezolana al calor de la Revolución cubana, pero los presidentes civiles de AD se responsabilizaron de las brutales campañas para derrotar a la izquierda armada, y en este proceso se ganaron el respeto y la lealtad de los militares. Las mismas guerrillas encontraron un escaso apoyo a sus esfuerzos entre los campesinos, que se veían beneficiados de un proceso extensivo de reforma agraria puesto en marcha por la coalición gobernante AD-COPEI en 1961. Políticamente aisladas y militarmente asediadas, las guerrillas terminaron su lucha armada a mitad de la década de 1960. Algunos ex-guerrilleros participaron en las elecciones de 1968, y el Presidente Caldera les garantizó la amnistía en 1969. Una división en el Partido Comunista en 1970-71 creó el MAS, que se sometió asimismo al proceso electoral para llegar al poder. Así, se neutralizó la amenaza desde la izquierda. En los años sesenta, tres facciones de $\mathrm{AD}$ se opusieron a una cerrada cooperación con COPEI y así cambiaron la fórmula desde el interior del partido, pero todas ellas fueron excluídas y después se convirtieron en irrelevantes en las próximas elecciones (Martz 1966). Tan tarde como 1968, algunos votantes, especialmente en las áreas urbanas, seguían apoyando a Pérez Jiménez u otros candidatos populistas derechistas, y el voto combinado por $\mathrm{AD}$ y COPEI decreció sobre las tres primeras elecciones. Pero en 1968, cuando Caldera consiguió la presidencia en una cerrada competencia y $\mathrm{AD}$ reconoció su victoria, resultó claro que la única alternativa realista para $\mathrm{AD}$ era $\mathrm{COPEI}$ (Myers 1973). En la próxima elección, los dos partidos consiguieron casi el 80 por ciento de los votos, y continuaron haciéndolo así durante los próximos quince años.

En 1970, además, el acuerdo Adecopeyano había arrinconado los cambios desde la izquierda, la derecha, y desde dentro, convirtiéndose en una fórmula muy efectiva para mantener la gobernabilidad. La legitimidad de esta fórmula fue alentada por la cuadruplicación de los precios del petróleo durante el gobierno AD entre 1974-79 de Carlos Andrés Pérez, quién tuvo la fortuna de ser elegido al comienzo del embargo petrolífero de la OPEP. La fantástica caída de los petrodólares facilitó para el acuerdo "comprar" el apoyo con patronazgo, subsidios a los consumidores, una moneda sobrevaluada, aumentos de salarios, y altas tasas de crecimiento económico.

\section{EL SURGIMIENTO DE LOS NUEVOS CAMBIOS EN LA DÉCADA DE 1980}

En la próxima década, sin embargo, la partidocracia venezolana desarrolló unas tendencias patológicas -una pérdida de dirección, una obsesión con el control y la corrupción. Se habían añadido nuevos términos a la fórmula de la gobernabilidad, demasiado vergonzosos para conocerse, pero sin duda muy reales. Estos se podrían describir como sigue:

1. Pérdida de dirección. En los veinte años que siguieron al Pacto de Punto Fijo, los gobiernos de AD y COPEI habían conseguido muchos de los objetivos políticos que sus partidos habían discutido a comienzos de los sesenta -reforma de tierra, nacionalización de la industria petrolera, expansión de la educación pública, creación de empleo, y la consolidación de la democracia. Si el debate en torno a la política hubiera continuado en el interior o entre los partidos durante esas dos décadas, habrían fijado nuevos objetivos para ellos mismos, pero no fue éste el caso: la tesis política de AD no se había renovado desde 1964. La disciplina partidista sofocó la expresión de las ideas controvertidas dentro de cada partido, y la concertación filtró la controversia fuera del debate intrapartidista. Con la amenaza de la expulsión, puesta de manifiesto en la serie de divisiones en el partido en los años sesenta, y además la amenaza del fin de la carrera política de la dirigencia partidista, pocos líderes del partido desearon sugerir nuevas ideas que podían provocar controversia. Los más peligrosos líderes del partido habían sido ya expulsados; los que permanecían en el partido eran aquellos que habían aprendido a mantenerse callados y es- 
perar lo que les dijera el liderazgo nacional. Aún más, tanto $\mathrm{AD}$ como COPEI habían virado hacia el centro, y cuanto más parecidos llegaron a ser, menos cuestiones sustantivas tenían que debatir. Las campañas presidenciales descansaban cada vez más en ataques personales, injurias, y esloganes biensonantes aunque sin sentido. Fue duro para los votantes apoyar a los partidos como medios para lograr algún fin honorable cuando, cada vez más, ellos se convirtieron en un fin en sí mismo.

2. Corrupción. Venezuela nunca se vió completamente libre de corrupción, ni siquiera durante los primeros años de gobierno democrático cuando el gobierno estaba persiguiendo al dictador anterior por corrupción. Pero dos hechos provocaron un aumento de la corrupción a finales de los años setenta -la bonanza petrolífera y la partidocracia. Como ha señalado Terry Karl, los beneficios conseguidos con el petróleo durante el gobierno de Pérez (1974-79) fueron superiores al 54 por ciento, en términos absolutos, que aquellos recibidos por todos los gobiernos venezolanos juntos desde 1917 (Karl 1982, 17). En este increíble diluvio de riqueza fue inevitable que algunos cargos públicos desviaran parte de este flujo hacia sus propios bolsillos, y que la responsabilidad financiera fuera inexistente. Lo que es más difícil de entender es por qué las prácticas de corrupción continuaron floreciendo incluso aún después de que el país se endeudara profundamente y cayeran los precios del petróleo, abocando al país a la crisis económica. La continuación de la corrupción requirió un clima de impunidad, que fue un subproducto de la partidocracia. Las cortes, incluso la burocracia, las universidades y la mayor parte de las otras instituciones, se politizaron ampliamente a través de las líneas partidistas y nunca parecían encontrar suficientes evidencias para justificar un juicio o una condena. Tuvo también que existir complicidad entre $\mathrm{AD}$ y $\mathrm{COPEI}$, porque ambos se comportaron como si existiera una claúsula secreta en el Pacto de Punto Fijo que prohibiera la persecución de la corrupción. La práctica de la concertación, que intentó moderar el conflicto político, sirvió igualmente para ocultar abusos de poder por parte del acuerdo Adecopeyano. Los practicantes de la impunidad no dudaron en racionalizar sus acciones sobre la creencia de que el descubrimiento pleno de la magnitud de la corrupción haría peligrar el conjunto del régimen democrático; retrospectiva e irónicamente, ellos parecen haber estado en lo correcto.

3. Obsesión de control. En manos de un creciente número de militantes del partido sin principios, la dedicación de los fundadores del partido a moderar el conflicto se convirtió en una obsesión por el control de otros actores de la sociedad civil. Los gobiernos respetaron ampliamente la libertad de organización, pero para los partidos, la fundación de cualquier organización independiente significó una llamada a las armas. Se hicieron todo tipo de esfuerzos por cooptar su liderazgo. Si esta táctica tenía éxito, la organización se sometería a la disciplina del partido. Si era infructuosa, los activistas del partido se infiltrarían a veces secretamente en la organización, tomarían su control, y la someterían al partido. Y si todo esto fallaba, crearían una organización paralela con la misma misión y competidora de la organización independiente con la asistencia de seguidores del partido en el gobierno local, intentanto que fracasaran los independientes. Esta última táctica terminó siendo tan frecuente que el término paralelismo se utilizó para describir el proceso. Al principio, los partidos tuvieron éxito en mantener su control, pero aquí y allí las organizaciones independientes ganaron pie -los sindicatos en el Estado de Bolívar, algunas asociaciones vecinales en las ciudades, y a finales de la década de 1980, algunas ONG's de derechos humanos y ecología (Silberberg 1991). Tales movimientos sociales deberían haber sido bien recibidos porque representaban un fortalecimiento de la sociedad civil y no significaban una amenaza mayor para la gobernabilidad que las comunidades de base cristianas en Brasil, o las coordinadoras de campesinos en México o las madres de la Plaza de Mayo en Argentina. Pero más que la bienvenida, fomenta esta sociedad civil floreciente y abre el sistema a una participación más genuina, los partidos trataron a estos grupos independientes como amenazas para el control partidista. Se perdió así una oportunidad para profundizar la democracia en Venezuela, y las organizaciones independientes respondieron vinculando sus demandas a una agenda antipartidista y antiestablisment.

Durante los años ochenta, los nuevos cambios en la partidocracia ganaron fortaleza en la misma medida en que se debilitó la gobernabilidad. El declive económico entre 1979 y 1990 actuó como catalizador para la oposición al acuerdo (Castro Escudero 1992). Cuando la crisis de la deuda estalló en 1983 y cuando cayeron los precios del petróleo, especialmente después de 1985, la capacidad de los partidos para controlar a la sociedad civil disminuyó. Se dispuso de menores recursos para el patronazgo o simplemente para afrontar las obligaciones rutinarias de los estados; los servicios públicos decayeron y la infraestructura comenzó a deteriorarse. Los partidos perdieron parte de su capacidad para cumplir sus promesas, para cooptar nuevas organizaciones -particularmente las asociaciones vecinales que surgieron al clamor de mejores servicios públicos- y ofrecer trabajos en el gobierno para los amigos y (antiguos) enemigos(4). En la medida en que disminuyeron los niveles de vida, creció el desencanto, lo que hacía más amargo el reconocimiento de que el país había sido tremendamente rico y lo había dilapidado. Durante la mayor parte de la década, sin embargo, la mayoría de los venezolanos estuvieron deseando canalizar su descontento en el proceso electoral como lo habían hecho durante años, conducido por la alternancia en el poder de AD y COPEI.

Durante el segundo gobierno de Pérez (1989-1993), dos hechos transformaron la ira antiparticipativa en una ira antiestablishment. En primer lugar, la política económica del gobierno de Pérez fue muy desilusionante. Mucha gente votó por Pérez en 1988 esperando que de alguna manera Venezuela volviera a retomar el boom que había disfrutado durante su primer gobierno, y la campaña de Pérez hizo más bien poco para desestimular esa esperanza. Por ejemplo, en

(4) Existe evidencia de que la provisión de agua y electricidad se incrementó entre 1981-89, lo que significa que esa preocupación por el deterioro de los "servicios públicos" se centró mayoritariamente en el aumento del crimen violento y en los recortes de los bienes y servicios esenciales (Templeton 1992) 
una encuesta llevada a cabo en enero de 1989 , justo antes de la elección, se mostró que el 45 por ciento de los venezolanos creían que su propia situación mejoraría al final del gobierno de Pérez, y la tasa de aprobación del presidente electo alcanzó el 79 por ciento (Myers 1992, 4-5). Pero uno de los primeros actos de Pérez como Presidente fue anunciar un drástico paquete de medidas de ajuste estructural, que incluían muchos aumentos de precios, sin una explicación suficiente de su necesidad. El día en que se pusieron en marcha, un sentimiento generalizado de traición y desesperación explotó en tres días de saqueos y revueltas conocidos como el Caracazo. El pueblo había cifrado sus esperanzas en una elección y cambio de gobierno, y sólo parecía que las cosas empeoraban. A corto plazo, esto era verdad: 1989 fue el año con peores resultados económicos desde la Gran Depresión, con una caída del 8,3 por ciento de la producción y una inflación que alcanzó el 80 por ciento. Para mayo de 1992, entre los dos intentos de golpes de estado, sólo el 28 por ciento creía que su situación mejoraría al final del gobierno de Pérez, y un 69 por ciento desaprobaba al presidente (Myers 1992, 4-5).

A largo plazo, las políticas de Pérez fueron responsables de una dramática recuperación económica que comenzó en 1991 , justo antes de que se produjera una segunda manifestación de la ira popular contra el conjunto de la clase política. En el período 1989-90, la prensa cada vez más independiente ofreció una constante y documentada cobertura de las actividades de corrupción que se habían producido durante la administración precedente. Se dieron frecuentes revelaciones sobre cómo las agencias de intercambio exterior RECADI habían intentado ofrecer beneficios ilegales a políticos y hombres de negocios con conexiones con el anterior Presidente Jaime Lusinchi (1984-89) y su secretaria y señora Blanca Ibáñez (con quién más tarde se casó). Cuando los venezolanos, sumergidos en las profundidades de la crisis económica, fueron bombardeados con reportajes sobre cómo habían desaparecido millones de dólares, llegaron a la inexplicable conclusión (aunque ciertamente exagerada) de que estaban sufriendo porque los políticos habían robado al país sus riquezas (Santana 1992). Por ejemplo, cuando una encuesta de 1984 preguntaba a los venezolanos por el factor que más había contribuído a la gran deuda externa del país, las dos respuestas más comunes fueron que "la mala administración de los fondos nacionales" (36 por ciento) y la "corrupción administrativa" (33 por ciento). De forma similar, en 1985 a la respuesta sobre las causas de la crisis económica, el 86 por ciento asignó "mucha responsabilidad" a la corrupción, mientras que el 74 por ciento lo hizo a la "mala administración de los recursos nacionales" y un 50 por ciento a la "caída de los valores morales" (Templeton 1992).

A pesar del continuo escándalo de tráfico sólo se castigó a una pequeña parte; Lusinchi e Ibáñez estaban todavía libres en el momento de escribir este artículo. Esta síntesis en torno a la ira por la situación económica y la ira por la corrupción fue más fuerte que cualquier otro tema tomado separadamente. Fue incluso más descarada por el hecho de que ahora el gobierno está reclamando a todo el mundo sacrificios para poder pagar por estos crímenes. Y esta vez, ellos dirigían su ira a los dos partidos porque COPEI, liderado por Eduardo Fernández, dio su apoyo a las políticas económicas de Pérez. (Pérez siguió adelante con sus políticas a pesar de las apagadas protestas de la facción dominante de su propio partido, $\mathrm{AD}$, pero $\mathrm{AD}$ era culpable de todos modos). De esta manera el acuerdo Adecopeyano se convirtió en el responsable de la corrupción, la impunidad y la misma crisis económica.

\section{LA BÚSQUEDA DE UNA ALTERNATIVA VIABLE}

Inicialmente parte de esta ira se revolvió contra la misma democracia. Después de todo, era duro admitir donde terminaba el acuerdo y donde empezaba la democracia; ambos nacieron al mismo tiempo y crecieron juntos, y el acuerdo mismo vino a equipararse con democracia. Esto ayuda a explicar por qué los líderes de la intentona golpista de febrero de 1992 disfrutaban de tanta popularidad: la pérdida de este particular régimen "democrático" era interpretado por el 26-32 por ciento de la población como un pequeño precio a pagar para deshacerse de un presidente odiado (Templeton 1992). Pero el segundo golpe de estado, en noviembre de 1992, fue un punto de no retorno en la definición de una alternativa a la partidocracia. Sus portavoces no fueron los jovenes oficiales limpios, coherentes y patrióticos de febrero, sino revolucionarios sucios e incoherentes. La idea de ser gobernados por ellos atemorizó a parte del apoyo al golpe, y urgió la búsqueda de una alternativa democrática.

Esa alternativa se definió en dos etapas en los siguientes quince meses. La primera etapa fue la acusación de Pérez en mayo de 1993 y la elección de un presidente interino -Ramón J. Velásquez. Como correspondía a una figura de transición, Velásquez no era ni un militante partidista ni una figura anti-establishment. (Era uno de los muchos "independientes" venezolanos que nunca se habían afiliado a un partido pero que se sabía simpatizaban con uno de ellos; en su caso, AD, a causa de su cercana amistad con Betancourt). En realidad, la gobernabilidad aumentó durante el gobierno interino, a causa de una reforma fiscal, una nueva ley bancaria, y otras medidas urgentes que se habían ido retrasado hasta que el voto de la acusación se aprobó rápidamente con el apoyo de $\mathrm{AD}$ y COPEI, sabiendo que el presidente independiente sería más responsable que el mismo partido (Villalba 1993).

La segunda etapa se inició con las elecciones generales del 5 de diciembre de 1993. Por su parte, AD y COPEI trataron de definir una alternativa al acuerdo como un acuerdo AD-COPEI renovado. En 1988 una reforma electoral había instituído elecciones directas para los alcaldes y gobernadores, y las elecciones estatales de 1989 y 1992 habían comenzado una significativa revitalización del liderazgo partidista a nivel local y nacional (Brewer-Carías, 1991; Kornblith and Levine 1994, 33). Una nueva generación de Adecos y Copeyanos (así como también MASistas y unos pocos líderes de Causa $R$, un nuevo movimiento sindical) estaba construyendo una base de auténtico apoyo a todos los niveles y cambiando el dominio de los cogollos en sus partidos. 
La renovación de los partidos dio un paso significativo hacia adelante cuando dos miembros de esta generación ganaron sorprendentemente las nominaciones presidenciales por $\mathrm{AD}$ y COPEI. En $\mathrm{AD}$ el nominado fue Claudio Fermín, un antiguo alcalde de Caracas; y en COPEI, el gobernador Oswaldo Alvárez Paz de Zulia vino a imponerse desde atrás en las primeras primarias abiertas del partido a Eduardo Fernández y a otros importantes líderes nacionales. Dado que eran candidatos nominados oficialmente por AD y COPEI, sin embargo, y dado que ambos se identificaban con las políticas económicas de la administración de Pérez, se encontraban en desventaja frente al candidato principal, Rafael Caldera.

Como fundador de COPEI, firmante del Pacto de Punto Fijo, antiguo Presidente, y participante clave en todo el proceso de concertación de los 35 años anteriores, Caldera parecería uno de los candidatos menos beneficiados por todo el sentimiento anti-establishment, pero lo fue. Dos hechos permitieron mejorar su imagen política. En primer lugar, el electrizante discurso que hizo ante el Senado con posterioridad al intento de golpe de estado en febrero de 1992. En esa ocasión, con retrasmisión en directo para toda la nación, no sólo frenó en seco el intento de golpe de estado sino que expresó la frustración popular contra Pérez, sus políticas y sus políticos irresponsables tan convincentemente que fue inmediatamente reconocido como el principal portavoz de la oposición. Su segundo hecho fue separarse de su propio partido a principios de 1993 para presentarse a presidente como candidato independiente con el respaldo de MAS y de otros 16 pequeños partidos reunidos en torno a Convergencia Nacional. Esta fue la ruptura más dramática posible con el establishment, no sólo porque abandonaba (y fue expulsado) el partido que fundara, sino porque tales deserciones hubieran sido impensables en Venezuela. Caldera ganó la votación con el 30,45 por ciento de los votos, un 24 por ciento de los votos fueron para Fermín, Alvárez Paz obtuvo un 23 por ciento y el fundador de Causa R. Andrés Velásquez, consiguió un 22 por ciento.

\section{PERSPECTIVAS PARA EL GOBIERNO DE CALDERA}

Este es un momento crítico para la gobernabilidad democrática en Venezuela: el acuerdo Adecopeyano ha perdido protagonismo, por primera vez en 35 años, y una figura anti-establishment está buscando una nueva fórmula para gobernar. Su búsqueda esta sujeta a frustracción debido a: (1) su débil base de apoyo, (2) la fuerza potencial de la oposición; (3) la pérdida de confianza en las elecciones; (4) el conflicto con los gobernadores, (5) unos militares divididos y recelosos, (6) las difíciles relaciones con el trabajo organizado, y (7) un inseguro sector privado. Venezuela era ciertamente bastante menos gobernable durante el gobierno de Caldera que en los años setenta. Sin embargo, no había alcanzado algún extremo teórico de ingobernabilidad; había perdido simplemente todas las ventajas que solía distinguirla de sus vecinos. Para poner la situación en perspectiva, Venezuela se había "latinoamericanizado". Algunas comparaciones de determinados aspectos de la gobernabilidad en otros países latinoamericanos nos ayudarán a apreciar las perspectivas de Venezuela.

\section{BASE DE APOYO}

Con el 30 por ciento del voto, Caldera no tiene más de un mandato para gobernar. (De hecho, después de contabilizar el 43,8 por ciento de la tasa de abstención, él fue elegido sólo con el apoyo del 17 por ciento de los votos registrados). Su coalición de gobierno está compuesta del izquierdista MAS además de Convergencia Nacional, que juntos controlan escasamente un cuarto de los asientos en el Congreso. Para empeorar las cosas, Convergencia es una mezcolanza de dieciséis pequeños partidos que van desde la extrema izquierda a la extrema derecha, encarnados en unos pocos desengañados Adecos y Copeyanos. Algunos de sus miembros podrían comenzar a desengañarse de la oposición tan pronto como la tasa de aprobación de Caldera descienda, dejando al Presidente incluso más aislado políticamente. Este no parece interesado en formar una coalición mayor; de hecho, sorprendentemente existen pocos representantes de MAS y Convergencia en su gabinete. En su esfuerzo por distanciarse él mismo de AD y COPEI y de los tecnócratas "IESA Boys" del gobierno de Pérez, Caldera ha intentado sobrepasar tanto a los políticos conocidos como a la élite política, contando con un gobierno dominado por los tecnócratas de segunda fila.

Más que formar una coalición de partidos, Caldera claramente intenta lograr mayorías ad-hoc para iniciativas legislativas específicas, que van directamente desde el pueblo para presionar al Congreso. Esta estrategia no le sirvió bien durante su primera administración, cuando también rechazó formar una coalición a pesar de haber obtenido la presidencia con el 29 por ciento de los votos, siendo neutralizado por el Congreso durante el 70 por ciento de su tiempo en el gobierno (Coppedge 1994b). Él puede esperar que las cosas sean más fáciles esta vez porque la mitad de los diputados fueron, por primera vez en diciembre, elegidos como candidatos individuales más que como miembros anónimos de listas de partidos y además están más dispuestos a romper con la disciplina partidista. Esas esperanzas estarían bien fundadas si él fuera un Presidente muy popular como Fujimori, pero no es el caso. Si tratara de aumentar su popularidad, por ejemplo, llevando a cabo alguna acción dramática contra la corrupción, sus relaciones con el Congreso serían más fáciles por algún tiempo. Pero hasta que eso suceda, el destino de otros presidentes menos populares con similares y estrechas bases de apoyo -Belaúnde (en su primer mandanto), Febres Cordero, Velasco Ibarra, Sarney, Collor, Illia, Allende- presagia o bien un estancamiento o una pugna de poderes con el Congreso (Linz 1990, Mainwaring 1993). Caldera ha expresado ya su deseo de modificar la Constitución para conseguir el poder de disolver el Congreso. Este se resiste a dejar en sus manos un arma tan poderosa, pero el hecho de que Caldera lo quiera es ya perturbador.

\section{LA OPOSICIÓN}

Mientras AD y COPEI eran duramente castigados en las encuestas, todavía controlaban una mayoría de los escaños en el Congreso (Tabla 1). Una aritmética simple aclara que 
Caldera no puede crear una mayoría legislativa sin AD y COPEI. Pero en este momento ninguno de los dos partidos constituyen ni una gran ayuda ni un gran obstáculo para el gobierno dado que están divididos internamente. Tanto AD como COPEI están llenos de recriminaciones sobre quién es el responsable del fracaso electoral y quién es el más capaz de llevarlos nuevamente al poder. Si se mantienen los moldes del pasado, ellos conseguirán la unidad interna antes de la próxima elección, pero la lucha cuerpo a cuerpo podría durar varios años. Mientras tanto, no estarán en situación de practicar la concertación -o prestan su apoyo a Caldera o forman un bloque obstruccionista para expulsarle. La última vez, que tanto AD como COPEI estuvieron en la oposición fue en 1957, y firmaron un pacto para oponerse al gobierno militar. Esta vez, la firma de un pacto podría conducirles a lograr una mayoría opositora y al estancamiento. Existen precedentes de tales coaliciones de mayoría opositoras tanto en Venezuela -AD encabezó la coalición en la legislatura sobre Caldera en su primer gobierno- y Perú, donde APRA y el partido UNO de Odría cooperaron para estancar a Belaúnde entre 1963 y 1968.

Como la composición de su gobierno sugiere, Caldera no quiere gobernar con $\mathrm{AD}$ y COPEI de todos modos, porque no trata de buscar su cooperación y ellos no desean tampoco ofrecérsela en esos términos. Ciertamente algunos de sus votos procedieron de Copeyanos; pero mucha de su legitimidad descansaba en su distanciamiento del acuerdo. Si invitara a $\mathrm{AD}$ o COPEI a entrar en la coalición corre el riesgo de perder su apoyo. Si invitara a COPEI, probablemente demandaría las cabezas de Eduardo Fernández y Oswaldo Alvárez Paz como precio, y este es un precio que el partido no estaría dispuesto a pagar. En todas partes los líderes de la oposición en América Latina han preferido a menudo negarse a la cooperación con el gobierno para asegurar un pobre resultado y mejorar sus expectativas en la próxima elección. Esto no ha sido el modelo en el acuerdo en Venezuela, pero puede convertirse en la norma ahora que los partidos del acuerdo están en la oposición frente a un Presidente anti-establishment.

TABLA 1: ESCAÑOS EN EL CONGRESO POR PARTIDO, 1993

\begin{tabular}{|c|c|c|c|c|}
\hline & \multicolumn{2}{|c|}{ Cámara de Diputados } & \multicolumn{2}{|c|}{ Senado } \\
\hline & escaños & porcentaje & escaños & porcentaje \\
\hline $\mathrm{AD}$ & 56 & 27,9 & 18 & 34,6 \\
\hline COPEI & 54 & 26,9 & 15 & 28,8 \\
\hline Causa R & 40 & 19,9 & 10 & 19,2 \\
\hline MAS & 51 & 25,4 & 9 & 17,3 \\
\hline \multicolumn{5}{|c|}{ y Convergencia Nacional } \\
\hline Total & 201 & 100,0 & 52 & 100,0 \\
\hline
\end{tabular}

FUENTE: NotiSUr, 4 de febrero de 1994

\section{CONFIANZA EN LAS ELECCIONES}

A pesar de las reformas electorales, las elecciones han perdido parte de su legitimidad como único camino para llegar al poder. La abstención se ha triplicado con respecto a 15 años antes, a pesar del voto obligatorio, y las acusaciones de fraude electoral se han hecho frecuentes. Mientras los numerosos vuelcos y la fragmentación del voto entre los distintos partidos indicarían que las elecciones son limpias, muchos venezolanos han comenzado a sospechar que los partidos más grandes se dividen frecuentemente los votos de los partidos que no estan representados en la votación(5). López Maya (1994) documenta intentos poco satisfactorios de $\mathrm{AD}$ para arrebatar las elecciones de gobernadores a Causa R. Dos de las elecciones a gobernadores en 1992 tuvieron que repetirse de nuevo en 1993 para dirimir cuestiones acerca de su limpieza, y tanto Caldera como Andrés Velásquez reclamaron que $\mathrm{AD}, \mathrm{COPEI}$, y los militares habían conspirado para privarles de miles de votos en la última elección presidencial(6). Aunque estas pretensiones puedan o no ser verdad, representan un síntoma de un declive de la gobernabilidad. No obstante, el problema no es tan importante como en Nicaragua, El Salvador, Honduras, Paraguay u otras democracias menos consolidadas. Los regímenes democráticos tales como Chile y Colombia han sobrevivido por muchos años a pesar de las disputas ocasionales en torno a los resultados electorales, así este solo hecho no situaría a la democracia venezolana en un peligro inmediato.

\section{EL CONFLICTO CON LOS GOBERNADORES}

Los gobernadores de los 23 estados en Venezuela se encuentran en una posición de crear problemas al gobierno nacional dado que son políticos con una base de apoyo independiente tanto respecto al Presidente como al partido. Los conflictos con Pérez fueron frecuentes dado que los gobernadores fueron elegidos directamente por primera vez en 1989 la división de poderes entre los gobiernos federales y estatales era todavía poco clara y los procedimientos para resolver los problemas se habían improvisado cada vez que surgía una dificultad. Los conflictos se podrían intensificar durante la administración de Caldera a causa de que los gobernadores más efectivos -aquellos que fueron reelegidos en 1992- llegarán a ser políticos derrotados según se aproximan las elecciones a gubernaturas en 1995, y algunos de ellos cuestionarán la autoridad de Caldera al presentar sus candidaturas a Presidente. Su potencial para la ruptura no debería exagerarse, sin embargo, dado que sus recursos son bastante limitados, y porque los gobernadores independientes no parecen causar serios problemas de gobierno en otras democracias presidenciales del hemisferio -Argentina, Brasil y los Estados Unidos.
(5) Comunicación personal de Luis Gómez Calcaño, febrero de 1994

(6) De acuerdo a la Junta Electoral Suprema, los votos disputados no son suficientes para alterar los resultados finales. Latin America Weekly Report (13 de enero de 1994) 


\section{RELACIONES CON LOS MILITARES}

Caldera fue quizás el mejor candidato para apaciguar la rebelión de los militares jóvenes: en el segundo aniversario del golpe del 4 de febrero de 1992, prometió la libertad para los 70 oficiales rebeldes que permanecían en prisión en Venezuela e invitó a regresar a los 52 restantes en el exilio en Perú y Ecuador. Tales actos, sin embargo, sólo exacerbaron las tensiones en el seno de las fuerzas armadas entre los militares jóvenes y el alto mando y además crearon tensiones entre éste y el comandante en jefe. Caldera afirmó su autoridad cesando al Ministro de Defensa y a los Jefes de Servicios tan pronto como tomó el poder, pero este hecho creó un resentimiento mayor hacia el nuevo Presidente y nuevas divisiones en el seno de los militares. Algunos oficiales también se opusieron a las acusaciones de Caldera de participación militar en el fraude electoral y se muestran recelosos sobre su habilidad para gobernar en los próximos cinco años. Después de dimitir, el Ministro de Defensa cesante Radamés Muñoz León decía(7):

"Esta situación me ha enfurecido. Esta no puede ser la recompensa que recibimos por la lucha democrática que hemos sostenido en el seno de las Fuerzas Armadas. Lloro por mi gente porque no sé lo que le ocurrirá al país con un gobierno precario que fue elegido escasamente por el 8 por ciento de la población, o el 16 por ciento de los votantes potenciales, y cuyo primer acto fue romper una institución que está al servicio de la patria y no de los partidos políticos, personalidades, o intereses económicos y políticos".

Las divisiones en las Fuerzas Armadas no parecen ser tan profundas como aquellas que se dan tipicamente entre los militares bolivianos, argentinos o peruanos, pero son lo bastante profundas como para mantener la preocupación en torno a futuros golpes de estado como los de 1992 que puedan encontrar a Caldera tan aislado e impopular como lo era Pérez en ese momento.

\section{RELACIONES CON EL TRABAJO ORGANIZADO}

Caldera está predestinado a tener una agria relación con los sindicatos venezolanos. Por un lado, les ha prometido mucho, como candidato con una imagen populista y como autor de la Ley de Trabajo, que ha sido injuriada por el sector privado por ser demasiado generosa con los trabajadores. Pero por otro lado, el déficit fiscal en Venezuela hace imposible para el estado ofrecer a los trabajadores muchos de los beneficios que la Ley de Trabajo les ofrece. (Un indicador: los beneficios petroleros, que solían cubrir el 70 por ciento de los gastos públicos, ahora cubren sólament el 40 por ciento). Y cuando los sindicatos se desengañaron y enfadaron con Caldera no tuvieron forma de restringirlos a causa de que no tiene virtualmente conexión institucional con los sindicatos. En vez de eso, la mayoría de los sindicatos están vinculados con los partidos de la oposición - $\mathrm{AD}$,

(7) "Encuentro del Ministro de Defensa con su contraparte colombiano", El Universal, 28 de Enero de 1994, pp. 2-19; recogido en FBIS-LAT-020, 31 de Enero de 1994, p.68.
COPEI, y Causa $\mathrm{R}$ (un sector minoritario del trabajo organizado está afiliado a MAS y MEP, que forma parte de Convergencia, pero tiende a seguir las indicaciones de la Confederación de Trabajadores Venezolanos (CTV), que está dominada por $\mathrm{AD}$ ). Cuando $\mathrm{AD}$ ha estado en la oposición en el pasado, ha alentado a los sindicatos a la militancia, o bien creando dificultades al gobierno, o bien ganando credibilidad para sus demandas como partido social democrático (Coppedge 1993b). Existen indicios de que el movimiento sindical de $\mathrm{AD}$ está reafirmando su independencia frente al partido. Pero si los sindicatos son o no independientes, no tendrá razón para retener sus miembros para Caldera. El aumento de la actividad huelguística es además inevitable. No obstante, los niveles de huelga han sido siempre comparativamente más bajos en Venezuela, así que no parece que las centrales venezolanas sean tan problemáticas como sus contrapartes en países como Bolivia, Argentina, Chile o Perú.

\section{RELACIONES CON EL SECTOR PRIVADO}

El proceso de ajuste estructural de la economía se ha ajustado también a la relación política entre el Estado y el sector privado en América Latina. Muchas empresas que habían crecido en dependencia con el proteccionismo, subsidios estatales, y conexiones políticas encontraron dificultades para sobrevivir en una economía de mercado más abierta, y perdieron parte de su influencia política; otras empresas que habían recibido bien el proceso de competencia, aumentaron su influencia. Este proceso schumpeteriano de destrucción creativa aumentó el conflicto dentro del sector privado en las primeras etapas del ajuste, pero donde el proceso pudo avanzar a largo plazo, como en Colombia, Chile, Bolivia y México, las empresas más competitivas fueron dominantes y desarrollaron una relación mutuamente más satisfactoria y transparente con el Estado que permitió la gobernabilidad en la arena económica. En Venezuela la elección de Caldera interrumpió este proceso antes de que las empresas más competitivas se impusieran.

La elección de Caldera significó una interrupción a causa de que su campaña envió señales mixtas y vaguamente inquietantes. Algunos hombres de negocios estaban preocupados por su alianza con el MAS y los comunistas; otros estaban confundidos por las políticas inconsistentes defendidas por sus consejeros más próximos; otros todavía estaban molestos por las promesas electorales para renegociar los términos del servicio de la deuda y para defender un tipo de cambio fijo cuando las medidas para luchar contra la inflación no se habían discutido. Después de la elección, el apoyo de Caldera a los controles de precios limitados, la suspensión de la porción menor de la tasa de valor añadido, y la inexistencia de un plan claro para reducir el déficit fiscal aumentaba la inseguridad. Algunos de estos miedos fueron aliviados por el camino tomado inicialmente por Caldera, pero para entonces un nuevo miedo sobrepasó a todos los demás: el temor a un colapso financiero acarreado por el fracaso del Banco Latino.

El Banco Latino puede interpretarse como un remanente del sector privado no reformado -un banco que comerciaba 
con conexiones y corrupción. Era el segundo banco más grande y el segundo en crecimiento en Venezuela, pero su éxito se construyó sobre las relaciones políticas y la débil regulación que le permitió ofrecer unos tipos de interés inusualmente elevados, y sus esfuerzos por cubrir sus responsabilidades eventualmente degeneraron en un esquema Ponzi masivo. Cuando el esquema colapsó a mediados de enero de 1994, 1,5 billones de dólares en depósito -el 20 por ciento del mercado- estaban en peligro, lo que afectaba no sólo a un millón de pequeños depositantes sino también a los fondos de pensiones de Petróleos de Venezuela, la compañía nacional eléctrica, y las Fuerzas Armadas, y aún más escandaloso, a casi la mitad de los fondos disponibles del equivalente venezolano del FDIC. Pero lo mejor, con este fracaso, que se combinó con los problemas de otros bancos debilitados, requirirá una fianza de 5 billones de dólares(8). Lo peor, podía llevar a un colapso financiero general que enviaría a millones de ciudadanos enfurecidos a las calles. La salud del conjunto de la economía venezolana es probablemente mejor que aquellas de otras naciones latinoamericanas, pero esta crisis ha creado una profunda incertidumbre en torno al futuro económico del país a medio plazo, y además ha minado gran parte del camino hacia la gobernabilidad en la arena económica conseguido antes de 1993.

En resumen, las perspectivas de la gobernabilidad en Venezuela son escasas. Comparado con un pasado de gran gobernabilidad, la sociedad está más polarizada, la nueva coalición gobernante está fragmentada y dividida, y los partidos en el acuerdo anterior, han sido recientemente ubicados en la oposición, lo que parece ineficaz o poco deseable para ayudar al nuevo Presidente a consolidarse. Esto no significa que la democracia esté a punto de quebrar. Existe poco entusiasmo hacia un gobierno militar en el momento actual, y la mayor parte de los actores estratégicos están deseando dar a Caldera una oportunidad. Si su intento de definir una alternativa al acuerdo Adecopeyano fracasa, sin embargo, no puede desecharse un golpe de estado con éxito. Y mientras tanto, Venezuela puede esperar encontrar mayores síntomas de ingobernabilidad -huelgas y protestas, resultados electorales en disputa, conflictos entre el gobierno federal y los gobernadores, incertidumbre económica, y especialmente confrontación entre el Presidente y el Congreso.

\section{REFERENCIAS BIBLIOGRÁFICAS}

Alexander, Robert J. 1964. The Venezuelan Democratic Revolution. New Brunswick, N.J.: Rutgers University Press.

Brewer-Carías, Allan R. 1991. "La descentralización política en Venezuela: 1990, el inicio de la reforma". En Dieter Nohlen, ed., Descentralización política y consolidación democrática, Madrid y Caracas: Síntesis y Nueva Sociedad, pp. 131-160.

Castro Escudero, Alfredo. "Venezuela: La encrucijada de la

(8) "El colapso del Banco Venezolano amenaza el futuro de la nación", Los Angeles Times, 14 de febrero de 1994, p. A-1. democracia". Comercio Exterior, 42: 3 (marzo de 1992):244-251.

Coppedge, Michael. 1993a. "Institutions and Democratic Governance in Latin America". Ponencia presentada a la Conferencia sobre "Rethinking Development Theories in Latin America", University of North Carolina, Chapel Hill, marzo de 1993.

1993b. "Parties and Society in Mexico and Venezuela: Why Competition Matters". Comparative Politics (Enero).

1994a. Strong Parties and Lame Ducks: Presidential Partiarchy and Factionalism in Venezuela. Stanford: Stanford University Press (en prensa).

— 1994b. "Venezuela: Democratic Despite Presidentialism". En Juan J. Linz and Arturo Valenzuela, eds., The Crisis of Presidential Democracy. Baltimore: The John Kopkins University Press (en prensa).

Gil Yepes, José Antonio. 1981. The Challenge of Venezuelan Democracy. New Brunswick, N.J.: Transaction.

Karl, Terry Lynn. 1982. "The Political Economy of Petrodollars: Oil and Democracy in Venezuela". PH.D. dissertation, Stanford University.

_ 1986. "Petroleum and Political Pacts: The Transition to Democracy in Venezuela". In Guillermo O'Donnell, Phillippe C. Schmitter and Laurence Whitehead, eds., Transitions from Authoritarian Rule: Latin America, Baltimore: The Johns Hopkins University Press, pp. 196-219.

Kornblith, Miriam and Daniel Levine. 1994. "Venezuela: The Life and Times of the Party System". In Scott Mainwaring and Timothy J. Scully, eds., Building Democratic Institutions. Stanford: Stanford University Press (en prensa).

Levine, Daniel. 1973. Conflict and Political Change in Venezuela. Princeton: Princeton University Press.

1978. "Venezuela since 1958: The Consolidation of Democratic Politics". In Juan J. Linz and Alfred Stepan, eds., The Breakdown of Democratic Regimes: Latin America. Baltimore: The Johns Hopkins University Press, pp. 82-119.

Linz, Juan J. 1990. "The Perils of Presidentialism". Journal of Democracy, 1:1 (Invierno): 51-69.

López Maya, Margarita. 1994. "El ascenso en Venezuela de la Causa R". Ponencia a ser presentada en el XVIII Congreso Internacional de LASA, Atlanta.

Mainwaring, Scott. 1993. "Presidentialism, Multiparty System and Democracy: The Difficult Combination". Comparative Political Studies.

Martz, John D. 1966. Acción Democrática: The Evolution of a Modern Political Party. Princeton: Princeton University Press.

Myers, David J. 1973. Democratic Campaigning in Venezuela: Caldera's Victory. Caracas: Fundación La Salle. - 1992. "Perceptions of a Stressed Democracy: Inevitable Decay or Foundation for Rebirth", ponencia presentada en la conferencia de noviembre "Democracy under Stress", patrocinada por el North-South Center de la Universidad de Miami e INVESP, Caracas.

Santana, Elías. 1992. Remarks at May forum on "Venezuela: Recent Events and Future Prospects", pa- 
trocinado por el Center for Strategic and International Studies and CAUSA, Washington, DC.

Silberberg, Mina. 1991. "Change and Continuity in 'ExtraClientelistc' Politics: Alternative Organizations of the Venezuelan Poor". Ponencia presentada al Congreso Internacional de LASA, Los Angeles.

Templeton, Andrew. 1992. "The Evolution of Popular Opinion". Draft Paper presented at the October confe- rence on "Lessons of the Venezuelan Experience", Woodrow Wilson International Center for Scholars, Washington, DC.

Tugwell, Franklin. 1975. The Politics of Oil in Venezuela. Stanford: Stanford UniversitY Press.

Villalba, Julián. 1993. "Venezuela's Future: Outlook for Investment and Privatization", 16 September talk at the Council of the Americas, Washington, DC.

\section{RESUMEN}

En este artículo Coppedge analiza las perspectivas de gobernabilidad en Venezuela. Este país, una vez la democracia más gobernable en América Latina, es ahora una democracia muy frágil. Después de estudiar los principales elementos de estabilidad de la fórmula venezolana, conocida como partidocracia, en una perspectiva histórica, Coppedge concluye que estas perspectivas son bastante reducidas. La sociedad venezolana está ahora más polarizada, la nueva coalición en el gobierno -el gobierno de Caldera - se encuentra fragmentada y dividida, y el anterior acuerdo entre los partidos parece ineficaz o éstos no desean apoyar al Presidente. Pero esto no quiere decir que la democracia esté a punto de quebrar en Venezuela, y este hecho dependerá del intento de Caldera por definir una alternativa con éxito al acuerdo adecopeyano.

\section{ABSTRACT}

Coppedge analyzes in this article the prospects for democratic governability in Venezuela. This country, once the most governable democracy in lati America, i now a very fragil democracy. After studying the main elements of the stability of Venezuela's formula, known as partyarchy in an historical perspectiva, Coppedge concludes that these prospects are poor. Venezuelan society is now more polarized, the new governing colaition - Caldera's government - is fragmented and divided, and the former establishment parties seem either unable or unwilling to help the new Presidente succeed. But this does not mean than democracy is abot to break down in Venezuela, and this fact will depend on Caldera's attempt to define a successful alternative to the Adecopeyano establishment. 